POS PROCEEDINGS

\title{
Third-generation SUSY Searches at ATLAS and CMS
}

\author{
Mark Hodgkinson ${ }^{a, *}$, on behalf of the ATLAS and CMS collaborations \\ ${ }^{a}$ University of Sheffield \\ E-mail: m.hodgkinson@sheffield.ac.uk
}

A number of searches for $\tilde{t}$ and $\tilde{b}$ pair production performed by the ATLAS and CMS collaborations at the LHC are discussed. These cover zero, one and two lepton final states as well as final states with Higgs or $Z$ bosons. Exclusion limits are placed using simplified models of $\tilde{t}$ and $\tilde{b}$ production.

online

*Speaker 


\section{Introduction}

Supersymmetry (SUSY) predicts the existence of a new family of particles. If natural SUSY exists, then gluino $(\tilde{g})$ and stop $(\tilde{t})$ particles have masses typically larger than the electroweak SUSY partner particles. Both ATLAS [1] and CMS [2], using proton-proton collisions at the LHC, have searched for both $\tilde{t}$ and sbottom $(\tilde{b})$ production in a variety of final states discussed here.

\section{Search for Stop and Sbottom Pairs in Zero Lepton Final States}

The ATLAS analysis targets the decay to a top quark and neutralino, $\tilde{t} \rightarrow t \chi_{0}^{1}$ [3]. ATLAS requires a lepton veto, jets, $b$-tagged jets and high $E_{\mathrm{T}}^{\text {miss }}$. Four signal regions (SR) A, B, C and D probe different regions of the $\tilde{t}-\chi_{0}^{1}$ phase space. SR D makes use of track jets which are sensitive down to a $p_{\mathrm{T}}$ of $5 \mathrm{GeV}$ [4], whilst SR A makes use of reclustered small radius jets for finding boosted $t$ production [5]. Large backgrounds are estimated using control (CR) and validation (VR) regions in this and all other analyses discussed. $Z \rightarrow v v$ is the dominant background in all SR, except for SR C (dominated by top quark pair production $(t t)$ ). Other backgrounds include production of $W$ bosons in association with heavy flavour jets, production of a single top (st) quark in association with a $W$ boson and $t t$ production in association with a $Z$ boson. No significant excess is seen in any SR and the $\tilde{t}$ mass is excluded up to around $1275 \mathrm{GeV}$ for a massless $\chi_{0}^{1}$ (Figure 1 (a)).

CMS has performed a search for both $\tilde{t}$ and $\tilde{b}$ pair production [6]. The analysis requires at least 2 jets and vetoes the presence of leptons, isolated tracks and photons. Cuts are applied on $H_{\mathrm{T}}, H_{\mathrm{T}}^{\mathrm{miss}}$ (both constructed from jets only) and $\Delta \Phi\left(H_{\mathrm{T}}^{\mathrm{miss}}\right.$, jet $\left.{ }_{1-4}\right)$. SR are binned in the number of jets, $b$-tagged jets, $H_{\mathrm{T}}$ and $H_{\mathrm{T}}^{\text {miss }}$. Backgrounds consist of $t t, s t, W / Z$ bosons in association with jets ( $W / Z+\mathrm{Jet}$ ) and multijet events. For a massless $\chi_{0}^{1}$ CMS excludes both $\tilde{t}$ and $\tilde{b}$ masses up to around $1200 \mathrm{GeV}$ (Figures 1(b) and (c)).

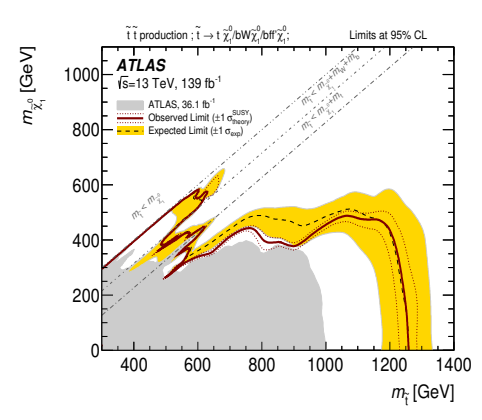

(a)

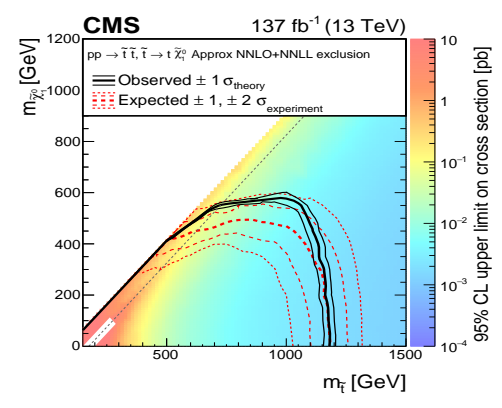

(b)

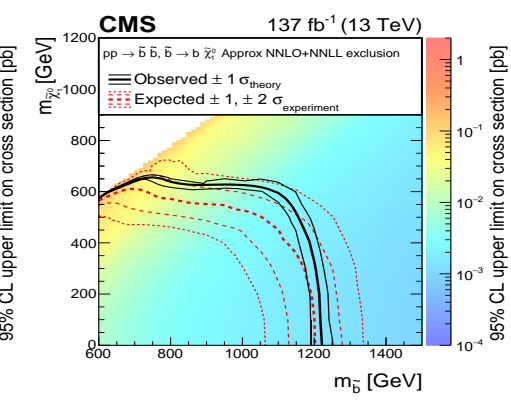

(c)

Figure 1: Results for the zero lepton final state: ATLAS exclusion in the $\tilde{t}-\chi_{0}^{1}$ plane [3] (a), CMS exclusion in the $\tilde{t}-\chi_{0}^{1}$ plane [6] (b) and CMS exclusion in the $\tilde{b}-\chi_{0}^{1}$ plane [6] (c).

\section{Search for Stop Pairs in One Lepton Final States}

Both ATLAS and CMS have performed analyses targeting $\tilde{t}$ pair production in one lepton final states [7, 8]. In the boosted region ATLAS (CMS) requires at least 4 (2) jets, at least one $b$-tagged jet, as well as cuts on $t$ reconstruction and other variables. In the intermediate region both ATLAS and CMS require at least 5 jets, one of which must originate from initial state radiation. ATLAS then requires at least 2 $b$-tagged jets, as well as cuts on $E_{\mathrm{T}}^{\text {miss }}$ and other variables. CMS requires at least one $b$-tagged jet and that the following inequality is satisfied $-p_{\mathrm{T}}^{\text {lepton }}<\max \left(50,250-100 \Delta \Phi\left(E_{\mathrm{T}}^{\text {miss }}\right.\right.$, lepton $\left.)\right)$, where $p_{\mathrm{T}}^{\text {lepton }}$ is the transverse momentum of the lepton and $\Delta \Phi\left(E_{\mathrm{T}}^{\text {miss }}\right.$, lepton $)$ is the difference in the azimuthal angle of the $E_{\mathrm{T}}^{\text {miss }}$ and lepton 2-vectors. The main backgrounds are $t t, t$ production in asociation with a vector boson and 
$W+$ Jet. The $s t$ and diboson backgrounds are also relevant. No significant excess is seen and hence exclusion limits are set for a variety of scenarios in the $\tilde{t}-\chi_{0}^{1}$ plane (Figures 2 (a) and (b)). Both experiments exclude $\tilde{t}$ masses up to around 1100 or $1200 \mathrm{GeV}$ depending on the decay modes considered.

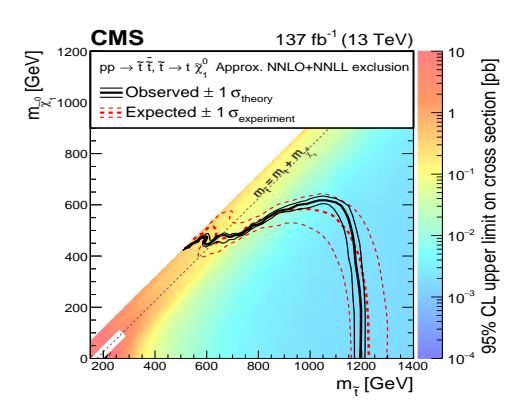

(a)

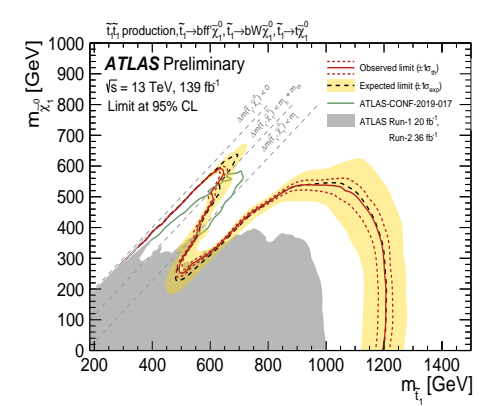

(b)

Figure 2: Results for the one lepton final state: Exclusion in the $\tilde{t}-\chi_{0}^{1}$ plane with a $100 \% \tilde{t}$ branching ratio to $t$ and $\chi_{0}^{1}$ with the CMS experiment [8] (a) and with the ATLAS experiment [7] (b). Other decay hypotheses can be found for CMS in [8].

\section{Search for Stop Pairs in Two Lepton Final States}

The CMS collaboration has performed an analysis targeting the decays $\tilde{t} \rightarrow t \chi_{0}^{1}$ and $\tilde{t} \rightarrow b \chi_{ \pm}^{1}$ with $\chi_{ \pm}^{1} \rightarrow W \chi_{0}^{1}$ or $\chi_{ \pm}^{1} \rightarrow v \tilde{l}^{ \pm}$, where $\tilde{l}^{ \pm} \rightarrow l^{ \pm} \chi_{0}^{1}$ [9]. Cuts are applied on the dilepton mass, the number of jets, the number of $b$-tagged jets and $\Delta \Phi\left(P_{\mathrm{T}}^{\text {miss }}\right.$, jet $\left._{1-4}\right)$. The SR are defined using the $P_{\mathrm{T}}^{\text {miss }}$ Significance $(S)$ and two definitions of the stransverse mass $\left(M_{\mathrm{T} 2}\right)$ [10] - one uses the leptons as the visible particles and the other the lepton and $b$-tagged jet pairs as visible particles. The SR are further subdivided by whether the lepton pair have the same or different flavours. In most SR the main backgrounds are $s t$ and $t t$, with mismeasured $P_{\mathrm{T}}^{\text {miss }}$ or a misidentified lepton. In the SR with high $M_{\mathrm{T} 2}$ cuts $t t$ pairs produced in association with $Z \rightarrow v v$ dominate. Exclusions are then placed in the $\tilde{t}-\chi_{0}^{1}$ plane for a variety of scenarios shown in Figure 3.

\section{Search for Stop Pairs in Final States with Higgs or $Z$ Bosons}

The ATLAS Collaboration has performed an analysis targeting the pair production of either $\tilde{t}_{1}$ or $\tilde{t}_{2}$ particles [11]. When $\tilde{t}_{1}$ are pair produced the analysis targets the decay $\tilde{t}_{1} \rightarrow t \chi_{0}^{2}$ with $\chi_{0}^{2} \rightarrow X \chi_{0}^{1}$ where $X$ is either a Higgs or $Z$ boson. When $\tilde{t}_{2}$ are pair produced the analysis targets the decay $\tilde{t}_{2} \rightarrow Z \tilde{t}_{1}$ with $\tilde{t}_{1} \rightarrow b f f^{\prime}$. Analyses requiring either one or three leptons are performed. In the former case a candidate $H \rightarrow b b$ decay is required as well as at least $4 b$-tagged jets, at least 4 or 6 jets, a transverse mass $\left(M_{\mathrm{T}}\right)$ greater than $150 \mathrm{GeV}$ and $S$ ( $E_{\mathrm{T}}^{\text {miss }}$ significance) greater than 7 or 12 . In the three lepton case one same sign opposite flavour (SFOS) lepton pair with mass within $15 \mathrm{GeV}$ of the $Z$ boson mass is required. Then SR are defined based on the number of jets, the number of $b$-tagged jets, the leading $b$-tagged jet transverse momentum $\left(p_{\mathrm{T}}\right)$, the $p_{\mathrm{T}}$ of the dilepton pair and $M_{\mathrm{T} 2}$ using the SFOS lepton pair and third lepton as the visible particles. The main backgrounds are $t t$ with heavy flavour jets or a $Z$ boson. Hadrons faking leptons and non-prompt leptons from $t t$ production are also relevant. No significant excess is seen and limits are set in the $\tilde{t}_{1}-\chi_{0}^{2}$ and $\tilde{t}_{2}-\chi_{0}^{1}$ planes (Figures 4(a) and (b)). ATLAS has also performed a search for $\tilde{b}$ pair production with a Higgs boson decaying to $b$ quarks [12] - the first full public likelihood is available for this analysis. CMS has also searched for $\tilde{b}$ pair production with a Higgs boson decaying to photons [13]. 


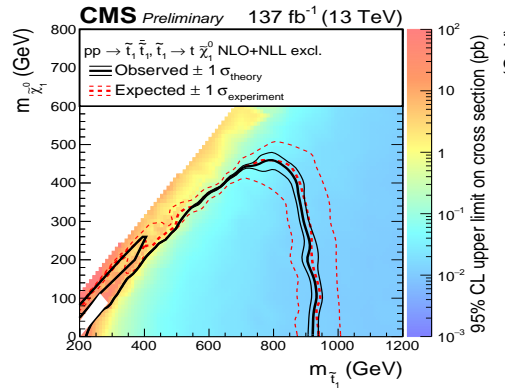

(a)

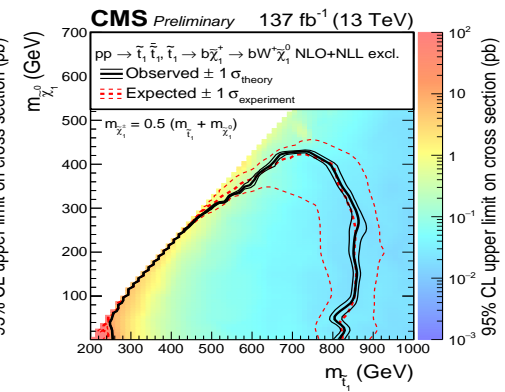

(b)

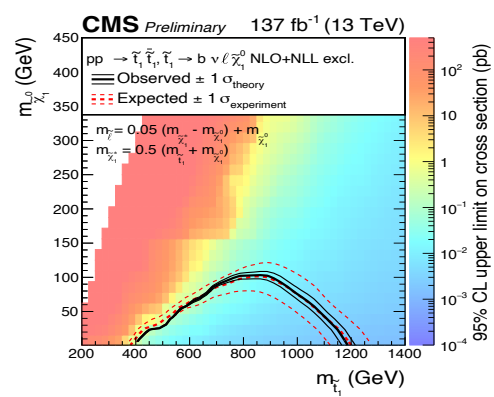

(c)

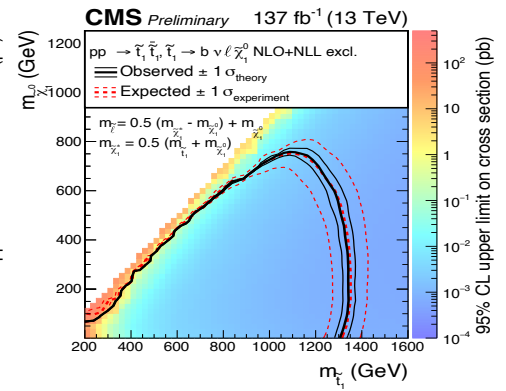

(d)

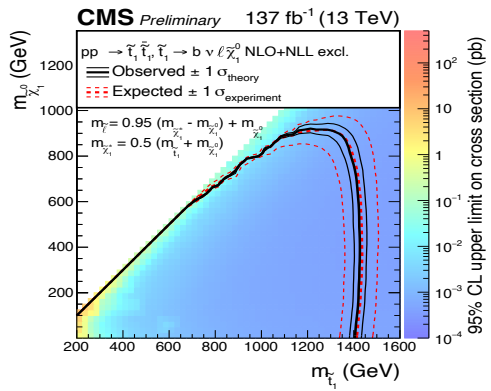

(e)

Figure 3: Results for the two lepton final state: CMS exclusion in the $\tilde{t}-\chi_{0}^{1}$ plane with a $100 \% \tilde{t}$ branching ratio to $t$ and $\chi_{0}^{1}$ [9] (a) and in the $\tilde{t}-\chi_{0}^{1}$ plane with a $100 \% \tilde{t}$ branching ratio to $b$ and $\chi_{ \pm}^{1}$ [9] (b). CMS exclusion in the $\tilde{t}-\chi_{0}^{1}$ plane for a $100 \%$ branching ratio of $\tilde{t} \rightarrow b v l \chi_{0}^{1}$ with the parameter $x$ set to 0.05 (c), 0.5 (d) or 0.95 (e) [9]. The parameter $x$ is defined by $m_{\bar{l}}=x\left(m_{\chi_{ \pm}^{1}}-m_{\chi_{0}^{1}}\right)+m_{\chi_{0}^{1}}$.

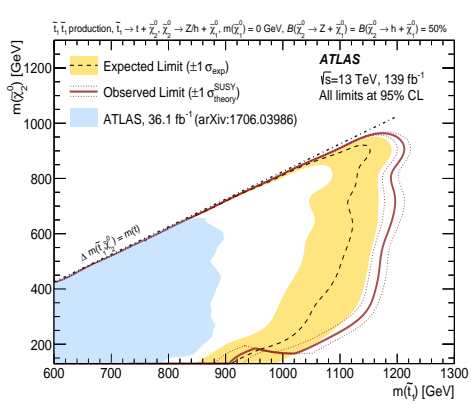

(a)

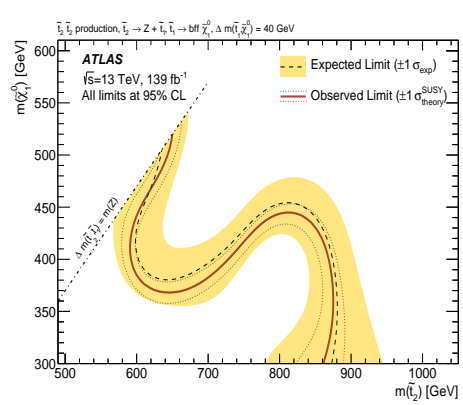

(b)

Figure 4: Results for final states with Higgs or $Z$ bosons: ATLAS exclusion in the $\tilde{t}_{1}-\chi_{0}^{2}$ plane [11] (a) and in the $\tilde{t}_{2}-\chi_{0}^{1}$ plane [11] (b).

\section{Conclusions}

Assuming a massless $\chi_{0}^{1}$ both ATLAS and CMS searches in the zero and one lepton final states have constrained the $\tilde{t}$ mass up to around $1200 \mathrm{GeV}$. The CMS zero lepton search also constrains the $\tilde{b}$ mass up to around $1200 \mathrm{GeV}$ and the CMS two lepton search constrains the $\tilde{t}$ mass up to between 800 and 1400 $\mathrm{GeV}$, depending on the model considered. The ATLAS $\tilde{t}$ pair search, including production of a $Z$ and Higgs boson, constrains the $\tilde{t}_{1}$ mass up to around $1200 \mathrm{GeV}$ assuming a $\chi_{0}^{2}$ mass of around $800 \mathrm{GeV}$. The ATLAS stop pair search, including production of two $Z$ bosons, constrains the $\tilde{t}_{2}$ mass up to around $870 \mathrm{GeV}$ for a $300 \mathrm{GeV} \chi_{0}^{1}$. 


\section{References}

[1] ATLAS Collaboration, JINST 3 (2008) S08003

[2] CMS Collaboration, JINST 3 (2008) S08004

[3] ATLAS Collaboration, Eur. Phys. J. C 80 (2020) 737

[4] ATLAS Collaboration, ATLAS-CONF-2019-027, https://cds.cern.ch/record/2682131

[5] ATLAS Collaboration, ATLAS-CONF-2017-062, https://cds.cern.ch/record/2275649

[6] CMS Collaboration, JHEP 10 (2019) 244

[7] ATLAS Collaboration, ATLAS-CONF-2020-003, https://cds.cern.ch/record/2711489

[8] CMS Collaboration, JHEP 05 (2020) 032

[9] CMS Collaboration, CMS PAS SUS-19-011, https://cds.cern.ch/record/2727987

[10] C.G. Lester and D.J. Summers, Phys Lett B 463 (1999) 99

[11] ATLAS Collaboration, arXiv:2006.05880

[12] ATLAS Collaboration, JHEP 12 (2019) 060

[13] CMS Collaboration, JHEP 11 (2019) 109 